\title{
L'exil de Thémistocle, ou L'expérience intime du sujet
}

\author{
ANGÉLIQUE CHRISTAKI Université Sorbonne Paris-Nord \\ PAULINE IAROSSI Université Paris IV-Sorbonne
}

RÉSUMÉ : Le mot «migrants» n'existait pas dans la langue des Hellènes; ils étaient plus familiers avec le vocable de l'exil et de l'asile. D'ailleurs, l'exil ne saurait se penser sans son corollaire, l'asile, comme le citoyen antique sans le barbare et la démocratie sans la tyrannie. À travers une métaphore située au carrefour de la fiction historique, de la philosophie politique et de la psychanalyse, cet article propose une réflexion sur l'exil comme condition fondamentale du sujet. L'exil est d'origine, car la langue de l'enfance, la langue dite maternelle est d'abord une langue étrangère. L'exil intime porte la marque d'une universalité qui fonde l'expérience subjective.

ABSTRACT: The word 'migrants' did not exist in the language of the Hellenes; they were more familiar with the terms 'exile' and 'political asylum.' Indeed, exile cannot be thought of without its corollary, political asylum, as the Ancient citizen cannot be thought of without the barbarian, and democracy without tyranny. Through a metaphor located at the crossroads of historical fiction, political philosophy and psychoanalysis, this article offers a reflection on exile as a fundamental condition of the subject. Exile is originary because the language of childhood, the so-called mother tongue, is originally a foreign language. Intimate exile bears the mark of a universality that founds subjective experience.

Mots clés : exil, asile, étrangeté, savoir, trahison, politique, tissage, féminin, langage

Dialogue 59 (2020), 403-414

(C) The Author(s), 2020. Published by Cambridge University Press on behalf of the Canadian Philosophical Association/I'Association canadienne de philosophie doi:10.1017/S0012217320000372 


\section{Thémistocle, figure d'exil}

Un texte ancien qui nous renvoie aux destins des premiers exilés dans le monde politique servira de canevas pour déplier une réflexion à partir des faits et des paroles qui datent d'il y a plus de vingt siècles. Nous y trouverons quelques éléments fondamentaux et donc diachroniques pour penser aujourd'hui la question de l'exil. À l'horizon de ces choses anciennes dont on parle généralement peu ou pas s'organise notre présent, notre présent politique auquel la question de l'exil est indéfectiblement liée. Les faits auxquels nous allons nous référer se déroulent en 480 avant notre ère, ils sont relatés par Plutarque ${ }^{1}$ et ils concernent la vie de Thémistocle, homme d'état et stratège athénien parmi les plus brillants de l'époque classique qui, exilé d'Athènes, se rend en terre barbare pour demander l'asile auprès d'Artaxerxès I.

Nous nous appuyons donc sur un épisode de l'histoire au déploiement tragique, qui pose l'exil ${ }^{2}$ en tant que constitutif d'un certain rapport à l'altérité et, de ce fait, comme un destin de tout temps pour l'être doué de parole. Voici un fragment de dialogue qui a lieu en terre d'exil entre Thémistocle, demandeur d'asile, et son hôte barbare Artaxerxès I, roi des Perses. Quand les deux hommes se rencontrent, Artaxerxès I, face à Thémistocle, «l'encourag [e] et le pri[e] de lui dire en toute franchise ce qu'il v[eut] à propos des affaires

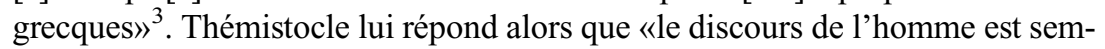
blable aux tapis bariolés : comme eux, une fois qu'il est entendu, il fait bien voir les sujets tandis qu'une fois replié, il les cache et les détruit; donc pour lui il faut du temps $[\ldots] \gg{ }^{4}$.

C'est en comparant le dépliement du discours de l'homme au dépliement d'un tapis que Thémistocle promet une parole sur les affaires grecques en échange de se voir accorder l'asile par le roi de Perse. Le tapis, en tant que produit d'un tissage, est évocateur à cet endroit de l'art du tisserand, l'art par excellence de l'homme politique ${ }^{5}$.

Ainsi, Thémistocle envisage de parler de ces affaires directement avec le roi et sans intermédiaire : il demande alors le délai d'une année pour apprendre à parler le perse dans le but de déplier dans la langue barbare les motifs de sa cause. En fonction du contexte, la question qui se pose est de savoir s'il s'agit d'une démarche de trahison, ou bien s'il s'agit d'une ruse de stratège permettant de gagner du temps. Il est à noter que l'exil se pose d'emblée comme une

\footnotetext{
Plutarque, Vies. Solon-Publicole-Thémistocle-Camille, tome II (2003).
}

2 À propos de la notion d'exil et du rapport à la démocratie, on pourra se référer, pour de plus amples informations, à Thucydide, Histoire de la guerre du Péloponnèse, tome I (1948). Voir aussi Plutarque, Vies parallèles, tome III, «Dion» (1950, chap. IV, 959d).

3 Plutarque (2003, 29, 3).

4 Ibid., 29, 4.

5 Voir Platon, Euvres complètes. Le politique, Paris, Les Belles Lettres (2003). 
épreuve d'entre-deux, en l'occurrence entre deux langues et entre deux cultures. L'exil est donc une affaire de langue et de culture ${ }^{6}$.

Cet extrait de dialogue place au cœur de la problématique de l'exil celle de l'art de tisser : c'est en évoquant l'art d'intercaler la chaîne et la trame - car tisser, «c'est bien, en somme, faire un entrelacement [...][,] l'art qui fabrique la chaîne et la trame» ${ }^{7}$ — que Thémistocle appuie sa demande d'asile. Cependant, l'art du tissage est pour le monde antique la métonymie ${ }^{8}$ de l'art politique, en tant que celui-ci est la fonction de la parole et du langage dans son exercice politique suprême au cœur de la cité des hommes, un art donc qui tisse la possibilité de vivre ensemble ${ }^{9}$. Or, l'art du tisserand trouve précisément son lieu d'origine dans le gynécée, dans le lieu des femmes, là où elles tissent le voile destiné non seulement aux affaires de l'oikos (aux affaires privées), mais également aux affaires du démos (les affaires publiques). Ainsi, le voile qui est tissé dans le gynécée est notamment destiné aux diverses cérémonies politico-religieuses de la cité, de sorte que l'art de tisser en tant que savoir-faire féminin évoque l'intime union du même avec l'autre, union fondatrice de l'art du politique, à savoir de l'exercice de la parole citoyenne dans la cité, un exercice exclusivement masculin, mais à l'origine d'un art féminin.

Cet extrait de dialogue entre le citoyen athénien exilé et son hôte barbare est supposé avoir lieu vers 470 avant notre ère, après que Thémistocle fut accusé de trahison par ses concitoyens grecs. Il s'exile donc et cherche l'asile en terre barbare, notamment auprès d'Artaxerxès I, qui est le fils de Xerxès I, roi de Perse. Artaxerxès était monté sur le trône après la mort de son père, ce roi qui fut vaincu en Grèce précisément par Thémistocle lors de la deuxième et dernière guerre médique, et notamment lors de la bataille de Salamine en 480, bataille fatale pour l'armée perse et humiliation personnelle pour Xerxès. Le passé des deux hommes, leurs affiliations et leurs cultures font que les questions de l'asile et de l'exil sont d'emblée inscrites dans la dimension tragique d'un entre-deux.

Il s'agit donc de remarquer que ce n'est pas auprès d'un inconnu que Thémistocle demande l'asile. Il ne s'est pas trouvé en terre barbare après une errance ni à la suite d'un exode. Son point de chute est une destination imposée par une réalité et précisément, relate Plutarque, par le fait qu'il redoute

6 Voir Heinz Wismann, Penser entre les langues (2012).

7 Platon, Pol. 208a.

8 À propos du fil et du tissage, souvenons-nous des événements qui précèdent la fondation de la démocratie à Athènes, et notamment de Kyloneion agos et des complices de Cylon qui, refugiés dans le temple de Minerve, attachèrent un fil à la statue de la déesse et, le tenant à la main, se mirent à descendre; mais le fil se rompit. Après cet événement commence avec Solon la fondation de la démocratie d'Athènes. Voir Plutarque (2003, Solon XII, 1-2, 194).

9 Voir Hannah Arendt, La politique a-t-elle encore un sens? (2007). 
davantage ses concitoyens athéniens qu'une vieille colère, fût-elle royale. Thémistocle exilé n'est ni un migrant ni un errant; il est ostracisé par vote démocratique dans l'Assemblé du peuple d'Athènes. Il s'avère alors que la question de l'exil est d'emblée politique ${ }^{10}$.

\section{Exil et trahison}

C'est d'ailleurs cette dimension du politique qui se perd dans le mot «migrant», qui est un mauvais choix dans la langue. Ce flux qui vient d'ailleurs, ces voyageurs forcés ne sont pas toujours des étrangers : il s'agit aujourd'hui de gens qui sont reconnus comme étant ceux qui n'ont rien d'autre que leur force de travail $^{11}$. La question qui se pose est alors de savoir : qu'est-ce que le lieu de la cité pour ceux qui sont assignés au rang des prolétaires?

Rappelons que le lieu de la cité, pour Thémistocle, est un lieu d'origine, et plus précisément le lieu de l'origine du citoyen. En ce sens, la cité constitue le lieu du politique à l'intérieur duquel prend corps la parole démocratique, la parole du citoyen.

Thémistocle est accusé de trahison, et l'exil se présente à lui comme la seule alternative susceptible de lui éviter le sort de Socrate et de tant d'autres hommes politiques de cette époque. Il est d'ailleurs remarquable qu'à l'époque classique, les hommes qui ont fondé la démocratie dans la cité d'Athènes, les réformateurs de la tyrannie, ceux qui ont donné à la démocratie non seulement son nom, mais l'une de ses formes les plus saisissantes, n'aient pas été exonérés du pire. Ainsi, ceux qui étaient considérés et reconnus par leurs concitoyens comme les meilleurs parmi les meilleurs dans la cité furent tous condamnés, exilés ou tués. De Solon, celui qui a écrit la première constitution à Athènes en vers, qui s'auto-exile lorsqu'il observe que le vent tourne pour lui, en passant par Aristide «le juste», par Thémistocle et jusqu'à Kimon, qui a été condamné à mort et tué, ayant évité de justesse le supplice sur la place publique, tous, sauf ce dernier, ont connu le même sort, à savoir l'exil pour cause de trahison.

10 Cornelius Castoriadis a longuement discuté cette question dans un article intitulé «La polis grecque et la création de la démocratie» (1986).

11 Par l'intermédiaire de la notion de plus-value, Lacan extrait l'affiliation prolétaire du sujet. Le sujet de Marx et celui de Lacan sont tous les deux aliénés et dépouillés d'un savoir. Ils sont à la place d'une vérité qui fait irruption comme symptôme. Marx fait du prolétaire le point d'insertion d'une vérité dans le système capitaliste, alors que Lacan, en prolongeant cette perspective, insiste sur la place du symptôme comme être de vérité. C'est en ce sens que le migrant contemporain n'est pas un esclave (au sens de cet esclave qui occupait avec son savoir le champ de la technè et qui dialoguait avec le maître antique). La dimension prolétaire du sujet est le symptôme d'un savoir duquel il est exilé, et qui dans le monde actuel est réquisitionné par un discours (dit capitaliste) qui ne fait pas lien et qui fait scission avec l'inconscient, le désir et l'amour. 
Et si tous ces hommes sans exception ont été aux prises avec l'exil, c'est parce que la parole de ces hommes dans la cité avait appelé à l'exil, une parole qui se donnait à entendre comme un risque mortel pour celui qui la profère comme pour celui qui l'entend.

De quel ordre est ce risque mortel? Quel est ce motif dans la parole qui fait appel à l'exil? Ce risque mortel est de l'étoffe de la trahison, mais cette trahison se déplie comme un motif dans le tissage politique de la parole citoyenne. Pour limiter le malentendu, précisions que ce motif de trahison ne se mesure ni comme un acte délictueux, ni comme l'indice d'une quelconque malhonnêteté commise dans la cité, ni comme un méfait de quelque ordre que ce soit. Plutarque écrit que pour les Athéniens, exiler quelqu'un après l'avoir accusé de trahison était une habitude. C'était, précise-t-il, une habitude avec tous les hommes dont la puissance pesait à leurs concitoyens, avec tous les hommes dont la puissance dépassait, à leur avis, la mesure d'une égalité démocratique. Or, l'habitude peut aussi être une question de jouissance ${ }^{12}$, une jouissance qui met à l'index une pesante démesure.

Ainsi, cette singulière trahison qui résonne comme une faute est en réalité un défaut de langage, un défaut de tissage politique que les Anciens avaient identifié comme une potentialité relative à la subversion de la cité des hommes, à savoir du lien politique, un défaut de langage qui signifie le détissage de l'étoffe démocratique de la cité, autrement dit le détissage de la possibilité de vivre ensemble. Cette potentialité avait trait à une jouissance particulière et inhérente à la parole citoyenne; en tant que telle, elle était inquiétante pour le citoyen et de ce fait passible d'exil.

La question qui se pose est de préciser : de quel ordre était-il, ce défaut susceptible de mettre la puce à l'oreille des Athéniens, d'indiquer que quelque chose clochait dans la cité, à savoir qu'une trahison était en puissance? De quel ordre était le défaut menant l'Assemblée du peuple à voter démocratiquement l'éloignement d'un citoyen hors des frontières de la cité?

De manière plus précise, cette trahison passible d'exil était l'affaire d'un dire qui transgressait une règle fondamentale de la cité : l'isègoria ${ }^{13}$. Dans le monde antique, la parole publique, et donc politique, était régie par cette règle spécifique. Le terme signifie littéralement «égalité de la parole dans l'agora»; il s'agit à la fois d'une égalité du temps de parole accordée aux citoyens et d'une égalité de la valeur citoyenne de la parole et de ses effets dans le lieu de la cité.

12 Nous entendons ici le mot jouissance comme un «au-delà du principe de plaisir» au niveau où Freud situe la force compulsive de la pulsion de mort; quelque chose se répète et il revient inéluctablement à la même place.

13 Cette notion d'isègoria est longuement discutée dans le texte majeur d'Isocrate, Sur la paix, tome III (1942, p. 12). 
La méfiance citoyenne à l'endroit de la transgression de l'isègoria portait sur toute parole privilégiée dont la brillance du dire serait susceptible de renverser l'ordre égalitaire et de subvertir ainsi le principe démocratique, l'ordre des égaux. «Chez nous personne ne doit être meilleur, mais si quelqu'un le devient que ce soit ailleurs et chez d'autres»» ${ }^{14}$. Voilà la cause et le verdict. Être meilleur signifie ne pas être égal. Mais de quelle égalité s'agit-il?

Si le temps d'une parole peut être compté, si une parole peut être équitablement partagée parmi les membres d'une assemblée, les effets de cette parole ne peuvent pas être mesurés d'avance ${ }^{15}$. Par conséquent, ce sont les effets non mesurables d'un dire excédant l'égalité des citoyens dans la cité qui constituaient pour les Anciens un défaut de citoyenneté. Ce défaut est en dehors de toute mesure, car il est exilé du lieu de la parole articulée, c'est un défaut de remémoration à l'intérieur même du tissage de la langue. Il émerge comme un défaut de mémoire et il résonne comme une faute à sanctionner.

C'est cet excès - au sens de l'hubris antique, excès pourtant inévitable et tenant à la structure — qui constituait pour les citoyens d'Athènes une faute de trahison passible d'exil. Si, pour les Anciens, la transgression de l'isègoria était considérée comme une menace pour la démocratie et un motif d'exil pour les citoyens, c'est parce que le régime de la parole citoyenne pouvait finir par coïncider avec sa propre négation : avec le régime de la tyrannie.

La transgression de l'isègoria rend ainsi compte d'un malaise spécifique qui concerne le destin du tissage politique de la parole citoyenne, à l'endroit précis où la langue démocratique contient la potentialité de son propre renversement politique, au point où celle-ci est susceptible de coïncider avec la langue tyrannique, à savoir avec un retour vers une insolite familiarité. La potentialité de ce renversement émerge comme quelque chose d'inquiétant et il est substantialisé comme une démesure en puissance, comme un retour possible vers la tyrannie.

C'est probablement le repérage de cette disposition unheimlich ${ }^{16}$ qui est à l'origine du spectre de trahison, une trahison qui s'opère dans la langue. Sur ce point, Freud développe un long commentaire dans son texte L'inquiétante étrangeté, où il suit à la trace l'évolution du mot heimlich dans la langue allemande. Ce qui ressort, c'est que parmi ces multiples nuances de signification, le petit mot heimlich en présente également une où il coïncide avec son contraire unheimlich. La signification du mot évolue en direction d'une ambivalence, jusqu'à ce qu'il finisse par coïncider avec son contraire. Ce qui est heimlich devient unheimlich ${ }^{17}$. À l'origine de cette ambivalence se trouvent le travail

14 Héraclite, Fragments. Citations et témoignages, trad. Jean-François Pradeau (2004, fragment 121).

15 Voir sur cette question la notion de parrêsia, qui prend une place importante dans le rapport entre parole performative et démocratie athénienne.

16 Sigmund Freud, L'inquiétante étrangeté et autres essais (1985 [1919]).

17 Ibid., p. 221. 
de la langue et notamment les résidus de la langue maternelle, les traces immémoriales et exilées du représentable que la langue maternelle dépose dans les mots. Il s'agit des résidus du narcissisme originaire qui se situent en deçà de toute représentation et qui pointent un réel inquiétant. Au-delà de la spécificité du mot heimlich, il s'agit d'entendre ce renversement de sens comme une opération de trahison à l'intérieur même de la langue. Ce renversement est un paradigme du travail de la langue et des conséquences de celui-ci chez tout être parlant. Il s'avère que ce renversement, avant d'être un événement politique dans la cité, est un événement de langue qui comporte la potentialité tragique du destin de la parole. Celle-ci est résonnance d'un réel qui laisse passer ce qu'aucun mot n'a le pouvoir d'éveiller; cette résonnance expose à tout ce qui est le plus étrangement intime à la parole, elle expose à l'ineffable, à ce qui est exilé de toute parole articulée ${ }^{18}$ et d'où s'origine la disposition inégalitaire du dire.

Ces résidus, exilés de la parole articulée, résonnent comme un défaut de mesure et ils pointent un réel vers lequel tend ce que Jacques Lacan appelle la mission de la langue. Dans ce contexte, Lacan se réfère à la langue maternelle, qu'il écrit en un seul mot : lalangue ${ }^{19}$. La langue maternelle définie par Lacan participe du singulier de la langue d'une mère et de l'universel d'une langue maternelle donnée ${ }^{20}$. C'est la langue dans laquelle un être humain a été élevé, c'est la langue transmise mais aussi la langue créée, langue qui permet au sujet une familiarité dans son usage à nulle autre pareille. Cependant, cette langue, qui offre à un sujet la capacité de se mouvoir en elle avec une certaine familiarité, admet également un réel indiscernable, un noyau indicible mais constitutif de la parole. Exilé du discours, ce noyau, tel le chant des Sirènes qui promettait une mort sans sépulture, peut être conçu à l'horizon des résidus de la langue qui transmettent une jouissance parfois mortifère, un excès de langue qui constitue la dimension «inégalitaire» de la parole.

Ce réel vers lequel lalangue tend est un destin inquiétant qui se substantialise comme possibilité d'un renversement politique là où la démocratie deviendrait le synonyme de la tyrannie. Une telle traîtrise s'origine dans le travail de la langue, et concerne le destin des mots. Il s'avère alors qu'une traîtrise s'opère en réalité dans la langue, suscitant une angoisse particulière qui a trait à un fantasme terrifiant et semblable à celui du retour dans le ventre de la mère ${ }^{21}$ : une mort sans sépulture évocatrice du chant des Sirènes.

18 Voir Angélique Christaki, La musique barbare de l'hallucination (2016).

19 Jacques Lacan, ... Ou pire, séminaire inédit (1971-1972).

20 J. Lacan, L'étourdit, dans Les Autres écrits (2001).

21 S. Freud, L'inquiétante étrangeté et autres essais (1985 [1919]). 


\section{Un étrange inquiétant à l'intérieur de la langue}

Ce réel ${ }^{22}$ de la langue qui excède le sens de la mesure et qui hante les citoyens de l'époque a trait à un non symbolisable, car à la fois impossible à dire et constitutif de la parole. Il s'agit d'un impossible à dire qui ne passe par les rets de l'imaginaire symbolisé; c'est donc cet objet insaisissable, insubstantifiable qui résonne comme cause de trahison et qui se déplie dans le tissage de la parole comme un motif et comme une cause d'exil.

Cet insubstantifiable renvoie à l'oubli, cet oubli auquel expose le chant des Sirènes. Le chant de ces anti-muses de la mort est en deçà des mots, sa dimension mortifère constitue une puissante résistance à la remémoration qui est la condition pour toute possibilité de travail de deuil.

En revanche, le tissage est un art d'emploké, c'est-à-dire qu'il est un art de liaison, une forme d'Éros au féminin. L'art de vivre ensemble qui a trait à l'art du tisserand s'origine dans un savoir-faire féminin, un savoir-faire hétérogène constitutif de l'art politique et du lieu de la cité des hommes et de leurs destins.

Pour ouvrir quelques pistes de réflexion, notons que le côté pour tous, celui de l'homme de la cité qui forge la citoyenneté, se tisse avec l'hétérité qui se signifie comme limite. Dans ce sens, la problématique est diachronique et elle fait résonner la question suivante : comment tisser la part phallique égalisatrice avec l'altérité du féminin, l'hétérité par excellence? Ainsi le savoir-faire féminin revient-il dans la trame du dire comme une question pour le savoir. Et c'est à cet endroit que le savoir se pose comme un défaut de savoir, un défaut qui est de l'ordre d'un trou, d'une faille dans la trame et qui n'est pourtant pas une simple faute de tissage. Ce défaut qui résonne comme une faute est constitutif du fait que toute parole est potentiellement une parole de trahison et de ce fait elle inclut l'exil en puissance.

Par conséquent, l'exil est constitutif du destin de l'être parlant, il fait partie d'un savoir-faire avec une altérité radicale qui opère dans la parole. Or ce savoir-faire se signifie dans la parole comme un défaut de tissage et il se traite comme une menace pour la cohésion de l'ordre établi. À ce propos, remarquons que Freud situe la menace pour la cohésion de l'ordre dans la cité des hommes du côté des femmes et qu'il se réfère à «l'hostilité des femmes à la civilisation» ${ }^{23}$. D'ailleurs le fait que les femmes entrent en opposition avec le

22 Chez Jacques Lacan, le Réel se noue avec le Symbolique et l'Imaginaire. Les théorisations de Lacan sur le réel évoluent tout au long de sa carrière. Dans son séminaire inédit, XXII RSI, à la séance du 10 décembre 1974, il dit : «On pourrait dire que le réel, c'est ce qui est strictement impensable [...]. Le réel a un statut particulier, du fait que l'on ne l'atteint pas». Le réel est inaccessible, il se distingue de la réalité, il a trait à la catégorie de l'impossible et de ce qui revient implacablement à la même place. On peut ajouter que cette notion se rapproche de ce que Freud situe dans un au-delà du principe de plaisir sans pour autant coïncider avec lui. 
courant de la culture et déploient leur influence retardatrice et frénatrice, ces mêmes femmes qui, au début, par les exigences de leur amour, avaient posé les fondements de la culture [explique le fait] que la femme se voit poussée à l'arrière-plan par les revendications de la culture et qu'elle entre avec celle-ci dans un rapport d'hostilité ${ }^{24}$.

Si alors on conçoit le langage comme l'habitat — le heim — de l'homme et de la femme, on voit que dans celui-ci gît aussi un élément hostile à la culture. Située par Freud du côté des femmes, cette hostilité, cette influence retardatrice et frénatrice finit bien par coïncider avec le mouvement fondateur de la culture, faisant ainsi de l'étoffe du langage le lieu où le féminin se déploie sur la crête d'une inquiétante étrangeté. Du même à l'autre se profile une trahison consubstantielle avec l'altérité radicale qui résonne comme une faille dans la cité des hommes, comme une potentialité de détissage des liens dans le langage et dans la parole; en somme, se dessine une destruction du lieu du logos.

\section{Du pouvoir politique du citoyen à l'impuissance du savoir}

De la cité des hommes, Thémistocle s'exile en terre barbare, mais en y allant il arrive directement dans la «gueule du loup» pour demander l'asile. C'est un fait que nous pouvons qualifier d'étrange au premier abord. On peut bien se demander ce que va chercher le citoyen auprès du roi barbare et de l'ancien ennemi, et ce que signifie cet asile auquel il fait appel.

Pour saisir ce curieux destin de l'exilé, il faut préciser qu'à l'époque, le barbare n'avait pas le même statut ni la même signification qu'aujourd'hui. La première signification du mot avait trait à la voix; «barbare» était le souffle de la voix dans son altérité radicale par rapport au langage et à la parole grecques. Dans l'Iliade, Barbarophono ${ }^{25}$ qualifie le souffle de la voix qui trahit à l'intérieur même de la langue grecque une provenance étrangère. C'est plus tard que le mot barbare acquiert le sens plus communément admis d'une langue autre que le grec.

Mais si le barbare a une connotation et un sens tout à fait spécifiques pour le citoyen antique, c'est parce qu'en réalité le barbare est la langue maternelle du grec. Les premiers Athéniens, selon Hérodote, appartenaient au peuple de Pélasges dont la langue maternelle était le barbare. Il s'agit alors de remarquer une structure mœbienne ${ }^{26}$ révélée par l'exil comme destin du retour au plus intime de la langue.

\section{Ibid., p. 41.}

25 Homère, Iliade, trad. Paul Mazon et al. (2004, chant 2, 867).

26 La bande de Mœbius est une figure topologique. Partis d'un point de surface, on arrive au bout d'un tour autour du trou central, à l'envers du point du départ, et il faudra un deuxième tour du trou pour revenir à la position initiale : la bande de Mœbius procède d'une topologie du double tour du trou. À travers cette figure, il s'agit de mettre l'accent sur le fait que la démocratie et la tyrannie ne sont pas des catégories opposées. Dedans-dehors, l'endroit où l'envers, voire moi-autre. Il 
L'exil est au principe un effet du mouvement du sujet dans sa parole et ce mouvement fonde l'altérité. Comme nous l'avons déjà évoqué, c'est Freud qui nous met sur la piste de cette réflexion en se référant au pouvoir de la langue de faire coïncider le sens des mots avec leur contraire, un pouvoir inhérent à la langue et susceptible de faire du lieu d'accueil un lieu d'origine et inversement.

Ce que les Anciens redoutaient était une parole révélatrice de trahison, une trahison porteuse d'une vérité à l'horizon d'un réel. Le tissage de la parole finissait par dévoiler une vérité qui devait bien rester voilée, à l'ombre, mais qui en est sortie. Ce dévoilement, comme l'indique $\operatorname{Lacan}^{27}$, ne fait que rappeler une nostalgie absolument douloureuse qu'il ne faudra jamais réveiller.

L'exil s'avère une épreuve de langue. L'entre deux langues n'est pas une affaire entre la langue d'origine et celle d'accueil, la langue tyrannique et la langue démocratique, la langue grecque et la langue barbare, puisque le voyage du sujet dans la langue est mœbien, qu'il s'opère à l'intérieur même de toute langue. C'est donc dans l'entre-deux d'une équivoque que prend racine le spectre de la trahison. À la base de toute équivoque se trouve l'origine sexuelle des mots, trace originaire de la langue qui comporte un savoir inaccessible dont tout être parlant est exilé. Point ombilical, pour toute parole articulable et exilée de celle-ci. Ce savoir insu est une altérité fondamentale, il est un parmi les noms de l'inconscient qui fonde la parole comme altérité et comme potentialité de trahison. Le dépliement de la parole - tel le déploiement d'un tapis - devient une affaire de trahison et les formations de l'inconscient, lapsus, oubli des noms propres, etc. constituent les motifs langagiers de cette trahison qui font de la parole une altérité. Cette altérité résonne dans la parole sans pour autant qu'elle puisse être substantialisable, évaluable, mesurable, égalitaire. Il s'agit d'un inquiétant défaut d'iségoria qui se donne à entendre dans la parole et qui fut très probablement passible d'exil chez le citoyen antique.

Cet aspect inquiétant provient d'un savoir étranger à lui-même, un savoir qui ne se sait pas; il est le spectre de la langue qui hante la parole et avec elle le lieu de la cité; il est en effet esprit de trahison. Or, ce spectre de langue qui hante le citoyen d'Athènes se soutient «d'un savoir sans pouvoir»; un tel savoir pose une question et la question est sans réponse, car il s'agit d'un savoir qui ne peut se donner comme réponse à quelque question que ce soit puisqu'il a pour fonction de sécréter la question et d'interroger la jouissance ${ }^{28}$.

(suite)

s'agit de les concevoir dans une forme de continuité (mœbienne), dans le sens où elles ne sont pas séparées par un bord, une forme de continuité sans franchissement.

J. Lacan, L'insu que sait de l'une-bévue s'aile à mourre, séminaire inédit (19761977, leçon du 11-01-1977).

28 Pour Lacan, la jouissance est, entre autres, cette substance qui irrigue le symptôme; elle fait partie de ce qu'interroge en dernier lieu une cure analytique. 
Un savoir politique sans pouvoir est le tissage en tant qu'étoffe du féminin et, si on ressert l'argument, un tel savoir pointe sa propre impuissance, son propre réel. C'est l'impuissance même du savoir qui constitue un aveu de trahison, puisqu'il fait résonner l'échec du savoir, un trou dans le tissage. Un savoir intraitable au sens de ce savoir qu'on ne peut pas traiter; un savoir qui trahit le savoir même. Un savoir au principe de l'exil inscrit dans la dimension de la langue.

\section{Références bibliographiques}

Arendt, Hannah

2007 La politique a-t-elle encore un sens? Paris, Éditions de l'Herne.

Castoriadis, Cornelius

1986 «La polis grecque et la création de la démocratie», Le Débat, n³8, p. 126-144.

Christaki, Angélique

2016 La musique barbare de l'hallucination, Paris, Hermann.

Freud, Sigmund

1985 L'inquiétante étrangeté et autres essais [1919], trad. F. Cambon, Paris, Gallimard.

Freud, Sigmund

1995 Malaise dans la civilisation [1929], trad. J. Odier, Paris, Presses universitaires de France.

Héraclite

2004 Fragments. Citations et témoignages, trad. J.-F. Pradeau, Paris, Flammarion.

Homère

2004 Iliade, trad. Paul Mazon et al., Paris, Les Belles lettres.

Isocrate

1942 Sur la paix, tome III, trad. G. Mathieu, Paris, Les Belles Lettres.

Lacan, Jacques

1971-1972 Ou pire, séminaire inédit.

Lacan, Jacques

1974-1975 RSI, séminaire XXII inédit.

Lacan, Jacques

1976-1977 L'insu que sait de l'une-bévue s'aile à mourre, séminaire inédit, leçon du 11-1-1977.

Lacan, Jacques

2001 L'étourdit, dans Autres écrits, Paris, Seuil.

Platon

2003 Euvres Complètes, trad. A. Diès, Paris, Les Belles Lettres.

Plutarque

1950 Vies parallèles, tome III, «Dion», trad. B. Latzarus, Paris, Classiques Garnier. 
414 Dialogue

Plutarque

2003 Vies. Solon-Publicola-Thémistocle-Camille, tome II, Paris, Les Belles Lettres.

Thucydide

1948 Histoire de la guerre du Péloponnèse, tome I, trad. J. Voilquin, Paris, Garnier Frères.

Wismann, Heinz

2012 Penser entre les langues, Paris, Albin Michel. 